\title{
MOVEMENT COORDINATION OF THE PELVIS IN A VIRTUAL GAME ENVIRONMENT
}

Foster RJ, Hawken MB, Barton GJ

Research Institute for Sport and Exercise Sciences, Liverpool John Moores University, Liverpool, L3 2ET, UK.

\section{SUMMARY}

Movement training specifically targeted at rotation of the pelvis may help to improve/overcome the primary component of pelvic retraction in patients with cerebral palsy $(\mathrm{CP})$. Healthy subjects when placed in a novel virtual environment provided evidence for a pre-established pattern of coordination, suggesting that wellpracticed core control cannot be improved over a short period of time through movement of the pelvis.

\section{CONCLUSIONS}

The results indicate that minimal learning occurred during testing, therefore future research will look at learning over a greater period of time. Further research on the differences in learning patterns of coordination between healthy subjects and CP patients will need to be established.

\section{INTRODUCTION}

Primary problems that exist in children with CP include loss of selective muscle control and muscular imbalance (Gage \& Novacheck, 2001) including the pelvic region, resulting in detrimental effects to everyday living tasks such as walking. Progression of the swing limb in normal gait leads to protraction and retraction on opposite sides of the pelvis. Abnormal pelvic retraction associated with $\mathrm{CP}$ hemiplegia improves in response to surgical intervention (Graham et al., 2005) but reduced movement control of the core remains a functional limitation. A virtual reality based game advocated by Barton et al. (2006), may improve movement coordination by focusing training on the pelvic region.

\section{PATIENTS/MATERIALS and METHODS}

Four healthy male participants (19-22 years) stood on a CAREN movable platform (MOTEK, Amsterdam, Holland) facing a video screen. The objective of the game was to burst balloons in a virtual environment by directing a "magic carpet" through three dimensional movement of the pelvis, where tilting the pelvis up/down and left to right controlled the direction of the carpet. A pre-determined trajectory was chosen that ensured pelvic rotation (PR) and tilt (PT) occurred simultaneously. In our definition a measure of coordination between PR and PT was the straightness of the carpet trajectory when approaching balloons, quantified as the cumulative difference between PR and PT for each trajectory. A $5^{\text {th }}$ order polynomial was used to illustrate the trends of coordination as it changed over time.

\section{RESULTS}

The trajectories for each subject show dissimilar patterns in coordination between PR and PT. Subject 1 showed a greater consistency in performing both simultaneously, represented by an almost constant difference in area between PR and PT ( $85 \%$ hit rate). Subject 2 produced a more variable pattern of coordination, showing slightly greater variability in the trend line, but still had a successful hit rate (85\%). Subject 3 controlled PR and PT better as he approached the target (93\% hit rate), highlighted by variability in the trend line towards the beginning of testing but an improvement in later trials. Subject 4 showed greater variability in co-ordinating PR and PT along the trajectory path (50\% hit rate). The trend line suggests subject 4 underwent the greatest period of learning during the session, with an improvement in producing PR and PT simultaneously over time.

\section{DISCUSSION}

The results suggest that every subject has their own pattern of coordination. In assessing the rate of learning the trend lines indicate that the majority of subjects show limited improvement in a short space of time and maintain a variable pattern of coordination throughout testing, except for subject 4 who demonstrated improvement. As the results show a general lack of improvement during testing, familiarisation before testing one's performance would not increase their level of core control they already possess. Further investigation will assess the period of time needed to provide an increase in performance level, and to assess the coordination of PR and PT for a larger population to understand how others adapt to the game. In patients with CP focus will be on how a person with reduced core control responds to training of coordination between PR and PT, and the pattern of learning patients produce in response to training.

\section{REFERENCES}

Barton, GJ et al. Gait and Posture 2006 24:101-102.

Gage JR, Novacheck TF. J Ped Orthop B 2001 10:265-74.

Graham HK et al. J Bone Joint Surg Br 2005 87:548-55. 